\title{
New Derivation of Simple Josephson Effect Relation Using New Quantum Mechanical Equation
}

\author{
Rashida Ismat Abdalrahman', Rasha Abd Elhai Mohammad Taha ${ }^{1,2 *}$, Isam Ahmed Attia1, \\ Mubarak Dirar Abd Allah ${ }^{1}$ \\ ${ }^{1}$ Department of Physics, College of Science, Sudan University of Science and Technology, Khartoum, Sudan \\ ${ }^{2}$ Department of Physics, College of Education, Majimaah University, Zulfi, KSA \\ Email: *r.taha@mu.edu.saisamattia7@gmail.com
}

Received 17 December 2015; accepted 4 March 2016; published 7 March 2016

Copyright (C) 2016 by authors and Scientific Research Publishing Inc.

This work is licensed under the Creative Commons Attribution International License (CC BY). http://creativecommons.org/licenses/by/4.0/

(c) (i) Open Access

\begin{abstract}
A relation of the Josephson current density equation is successfully derived; this is done through a new derivation of the equation of quantum by neglecting kinetic Newtonian term in the energy expression.
\end{abstract}

Keywords

Quantum Mechanics, Superconductors, Josephson Effect, Tunneling Current

\section{Introduction}

In quantum mechanics, it's well known that an electron can tunnel through a barrier, even if its kinetic energy is less than the barrier potential, thereby producing tunneling current. Josephson effect describes a tunneling in the superconductors, in which superconducting pairing between electrons is important [1]. This tunneling was predicted in 1962, by the British student Brian D. Josephson who predicted two effects which could experimentally verify shortly afterwards [2]. The modern Josephson voltage standard is based on one of these effects.

The Josephson effects occur if two superconductors are weakly coupled, e.g. by separating them by an insulating layer of a few nanometers in thickness.

Josephson effect is superconducting phenomenon. The phenomena of superconducting have no a full unique theory that describes all superconducting effects especially the high superconducting interaction.

Recently many nonconventional theories have tried to describe high superconducting phenomena; in one of them new Schrödinger equation based on plasma equation was used [3]. Some also were based on new conduc-

${ }^{*}$ Corresponding author.

How to cite this paper: Abdalrahman, R.I., Abd Elhai, R., Attia, I.A. and Abd Allah, M.D. (2016) New Derivation of Simple Josephson Effect Relation Using New Quantum Mechanical Equation. Natural Science, 8, 85-88.

http://dx.doi.org/10.4236/ns.2016.83011 
tion mechanism like polarons [4]-[6], but the new of them tried to simplify Josephson effect by simple theoretical treatment.

The derivation of Josephson Effect relationship is complex. Therefore one needs a simple derivation. This is done through a new derivation for the quantum equation in Section 2. Then this equation is solved for the Josephson relationship in Section 3; Section 4 and Section 5 are devoted to discussion and conclusion.

\section{New Quantum Equation}

The Newtonian energy $E$ is a sum of kinetic and potential energy $v$, i.e.:

$$
E=\frac{1}{2} m v^{2}+V=\frac{P^{2}}{2 m}+V
$$

where $m, v, p$ are the mass, velocity and momentum respectively. According to a theorem of Bloch's [7], in such superconductors the momentum $p$ is zero.

$$
p=\frac{m v^{2}}{2}+\frac{q A}{C}
$$

thus (1) becomes:

$$
E=V
$$

This is related to the fact that in Josephson effect the tunneling potential is considered to be larger than kinetic term squaring both sides yields:

$$
E^{2}=V^{2}
$$

Multiplying both sides by $\Psi$, one gets:

$$
E^{2} \Psi=V^{2} \Psi
$$

The wave function of a free particle is given by:

$$
\Psi=A \mathrm{e}^{\frac{i}{\hbar}(P x-E t)}
$$

Differentiating both sides with respect $x$ and $t$ twee

$$
\begin{gathered}
\frac{\partial \Psi}{\partial t}=-\frac{i}{\hbar} E \Psi \\
\frac{\partial^{2} \Psi}{\partial t^{2}}=-\frac{i}{\hbar} E \frac{\partial \Psi}{\partial t}=\frac{i^{2}}{\hbar^{2}} E^{2} \Psi=-\frac{E^{2}}{\hbar^{2}} \Psi \\
-\hbar^{2} \frac{\partial^{2} \Psi}{\partial t^{2}}=E^{2} \Psi
\end{gathered}
$$

Similarly:

$$
\begin{gathered}
\frac{\partial \Psi}{\partial x}=\frac{i}{\hbar} P \Psi \\
\nabla^{2} \Psi=\frac{\partial^{2} \Psi}{\partial x^{2}}=\frac{i P}{\hbar} \frac{\partial \Psi}{\partial x}=\frac{i P}{\hbar}\left(\frac{i P}{\hbar}\right) \Psi=\frac{i^{2} p^{2}}{\hbar^{2}} \\
-\hbar^{2} \nabla^{2} \Psi=P^{2} \Psi
\end{gathered}
$$

Substitute (6) in (4) to get

$$
-\hbar^{2} \frac{\partial^{2} \Psi}{\partial t^{2}}=V^{2} \Psi
$$

\section{Josephson Effect Equation}

In Josephson effect electrons are considered as having small kinetic energy compared to the potential. Thus 
Schrodinger Equation (8), in which kinetic term is neglected is suitable for describing the Josephson effect.

To derive Josephson effect equation, consider the solution

$$
\Psi=D \sin (\alpha t+\phi)
$$

The tunneling potential is constant inside a superconductor, thus

$$
V=V_{0}
$$

From (9), one can differentiate $\Psi$ with respect to time twice to get:

$$
\begin{gathered}
\frac{\partial \Psi}{\partial t}=\alpha D \cos (\alpha t+\phi) \\
\frac{\partial^{2} \Psi}{\partial t^{2}}=-\alpha^{2} D \sin (\alpha t+\phi)=-\alpha^{2} \Psi
\end{gathered}
$$

Substitute (10) and (11) in (8) to obtain:

$$
\begin{gathered}
+\hbar^{2} \alpha^{2} \Psi=V_{0}^{2} \Psi \\
\alpha^{2}=\frac{V_{0}^{2}}{\hbar^{2}} \\
\alpha= \pm \frac{V_{0}}{\hbar}
\end{gathered}
$$

By Substituting (12) in (9) and choosing a negative sign, that is in dealing with the change in potential energy one gets

$$
\Psi=D \sin \left(-\frac{e V_{0}}{\hbar} t+\phi\right)
$$

But the energy density $J$ is given by:

$$
\begin{gathered}
J=e \frac{\partial n}{\partial t}=e \frac{\partial|\Psi|^{2}}{\partial t}=2 e|\Psi| \frac{\mathrm{d}|\Psi|}{\mathrm{d} t} \\
=2 e D \sin (\alpha t+\phi)\left(-\frac{e}{\hbar} V_{0}\right) \cos (\alpha t+\phi) \\
=-2 \frac{e^{2} D V_{0}}{\hbar} \sin \theta \cos \theta \\
\theta=\phi-\frac{e V_{0} t}{\hbar}
\end{gathered}
$$

By using mathematical identity

$$
\sin 2 \theta=2 \sin \theta \cos \theta
$$

One can rewrite Equation (14) to be

$$
J=-\frac{e^{2} D V_{0}}{\hbar} \sin \left(2 \phi-\frac{2 e V_{0} t}{\hbar}\right)=A \sin \left(2 \phi-\frac{2 e V_{0} t}{\hbar}\right)
$$

Setting:

$$
2 \phi=\delta(0)
$$

The current density is given by:

$$
J=J_{0} \sin \left(\delta(0)-\frac{2 e V}{\hbar} t\right)
$$


Which is the Josephson effect equation.

\section{Discussion}

Equation (2) shows a new energy equation based on Newtonian mechanics, with the neglected kinetic term. This equation is used to derive a new quantum Equation in (8). This new equation is based on Newtonian energy with no kinetic term beside the wave equation of a free particle. This derivation resembles simple derivations of Schrodinger equation except the fact that the kinetic term is neglected

This equation is used to derive simple Josephson current density equation. This Equation (16) is the same as the old one, but derived using simple arguments.

\section{Conclusion}

Neglecting kinetic Newtonian term in the energy expression, one can easily derive a new quantum equation. This equation is shown to be successful in deriving simple Josephson current density equation.

\section{References}

[1] Josephson, B.D. (1962) Possible New Effects in Superconductive Tunnelling. Physics Letters, 1, 251-253. http://dx.doi.org/10.1016/0031-9163(62)91369-0

[2] Abd Elhai, R., Hilo, M.H.M., Abd Elgani, R. and Abd Allah, M.D. (2013) Using the Tight Binding Approximation in Deriving the Quantum Critical Temperature Superconductivity Equation. Natural Science, 5, 941-946. http://dx.doi.org/10.4236/ns.2013.58114

[3] Saxena, A.K. (2009) High-Temperature Superconductors. Springer, New York.

[4] Landau, L.D. and Pekar, S.I. (1948) Effective Mass of a Polaron. Zhurnal eksperymental'noyi i teoretychnoyi fizyky, 18, 419-423.

[5] Landau, L.D. (1933) The Movement of Electrons in the Crystal Lattice. Physikalische Zeitschrift der Sowjetunion, 3, 644-645.

[6] Pekar, S.I. (1951) Research in Electron Theory of Crystals, AEC-tr-555. US Atomic Energy Commission

[7] Tinkham, M. (1996) Introduction to Superconductivity. McGraw-Hill, New York. 